MARIO BUNGE

\title{
CORRECTIONS TO FOUNDATIONS OF PHYSICS: CORRECT AND INCORRECT
}

I am glad that Strauss [11] has chosen to waltz to the tune of my Foundations of Physics [2]. His overall evaluation of the book, as "an excellent piece of work" that "has a good chance to become a standard work of reference", is flattering, particularly for coming - as he puts it with characteristic modesty - from a fellow worker with "a longer and/or more detailed experience in this difficult field of research".

As to Strauss' criticisms, I found all of them interesting and worth being discussed, even those belonging to the popular mythology of physics. However, I agree with only 5 of his 21 critical remarks: I find 13 of them wrong and the last 3 irrelevant. I will presently give reasons for this $1: 4$ score. In order to ease the reader's task I will adopt my critic's format. I shall also pose some exercises and problems which, I hope, may prove instructive.

\section{MATHEMATICAL ERRORS}

1. Galilei, Lorentz and Einstein groups (p. 87). The critic is right. Strictly speaking, it is a question of replacing, not of including one group in another. However, one often does speak loosely of one group of transformations being included in a larger group if the transformation law that characterizes the latter is less demanding than the one that characterizes the former.

Exercise: Is it possible to characterize physical theories either by their transformation properties (e.g. general covariance) or by the number of universal constants they contain?

2. Spacetime (p. 184). The critic is wrong. The symbol ' $E{ }^{3} \times T$ ' designates both the Galilean and the Lorentzian spacetimes, because it stands for a 4-manifold that is Euclidean for any temporal cross-section. It does not specify the inner product - nor is it supposed to. The metric of SR is not postulated in my book but is gotten as a theorem (p. 191).

Problem: Is the Lorentz metric derivable from even weaker geometrical assumptions (e.g. affinity) conjoined with physical laws? 
3. Coordinate transformations (p. 219). The critic is right. Since coordinates are functions, coordinate transformations map functions into functions in such a way that the manifold itself is not affected. Spacetime is regarded as an external reality that can be mapped onto $R^{4}$ in any number of ways. Coordinate transformations are just changes of representation: they effect changes in the picture, not in its referent.

Problem: State the conditions under which, in the various fundamental theories, a coordinate transformation mirrors a possible change of frame.

\section{ERRORS IN LOGIC}

4. Transformation properties (p. 54). The critic is wrong. A statement like "Theory $T$ is covariant under the group G of transformations" is not a statement in $\mathrm{T}$ - hence not a statement concerning the referent(s) of $\mathrm{T}$ but a statement about $\mathrm{T}$ (and G): it is a metastatement. Moreover, it belongs to a purely mathematical theory concerning $\mathrm{T}$ : namely, to the transformation (meta)theory of $\mathrm{T}$ (e.g. the theory of unitary transformations). As a consequence, that statement is devoid of factual meaning: it talks about certain mathematical operations on the formulas of $T$, not about the things $T$ talks about. Hence, contrary to popular opinion, a statement like "Newton's laws of motion are Galilei covariant" is not a physical postulate (pace Strauss) but a metastatement. Consequently it can only be checked by performing the proper calculation, not by making any observations. Surely such metastatements may occur not only as formally proved metatheorems but also as heuristic guides during the theory construction stage - but this does not lend them any factual meaning $[3,4]$.

Exercise: Collect and analyze the metanomological statements in the basic physical theories and establish the relation between law invariance and process invariance.

5. SR-CEM relations (p. 62). The critic is wrong. SR presupposes CEM both logically and heuristically. Otherwise I would not have succeeded in deriving the Lorentz transformations from my postulates for SRK. Just consider the symbol ' $c$ ', which makes no sense but in electromagnetic field theories. To reinterpret ' $c$ ' in SRK as the limiting velocity of signals (as Strauss and many textbooks would have it) would make no sense unless special postulates regarding signals in general were added - but 
then SRK would become something else, namely a theory of signals, including acoustic signals. Yet SRK does not happen to be such a theory: for one thing it is not true of signals other than electromagnetic waves. Besides, the frame invariance of $c$ would be unintelligible without CEM: only this theory (and its quantal versions) accounts for the frameindependent behavior of the electromagnetic radiation field (in contrast to the field attached to the sources). Of course the fact that SR presupposes CEM does not mean that the latter entails the former without further ado. I need eight specific axiom groups, in addition to the set of presuppositions, to get SRK. The fact that SRK is so often expounded without making any reference to CEM does not prove that such expositions are logically sound and intelligible.

Exercise: Elucidate the syntactic, semantic and pragmatic concepts of theory presupposition.

6. Missing axiom (p. 164). The critic is wrong. One should not postulate the existence of inertial frames (whether Galilean, Lorentzian, or Einsteinian). For, if one did, then one would be claiming that the theory concerned holds in (relative to) at least one reference frame. And this is something for experiment, not for theory to decide. Indeed, the conjunction of my Df. 9 of electromagnetic inertial system with Strauss' proposed existence postulate entails that the axioms of CEM are true. Which, of course, is false.

Exercise: Give precise definitions of 'inertial frame' in various theories and find out their relations.

7. Logical miracle (p. 252). The critic is wrong. Any function satisfying a set of axioms for probability is a probability measure - by definition. Hence the probabilistic character of a given function need not be postulated: it may be found out (proved), as I have done it for the norm of the state vector in QM. This conclusion is reached by proving that the given function satisfies Kolmogoroff's axioms. The factual meaning of the function in question is secured by having listed (on p. 242) the physical probability theory, rather than the bare mathematical axioms of it, among the presuppositions of QM. There is no miracle here. Nor is it miraculous - even though it is not widely known - to obtain definite probability values from non-probabilistic premises. Thus the probable outcome of a run of roulette throws can be deduced from elementary kinematic hypotheses conjoined with very general mathematical assumptions 
concerning an arbitrary distribution of initial velocities (Khintchine [10]).

Exercise: Analyze the inference of probability statements from nonprobability statements with the help of the method of arbitrary functions.

\section{MISTAKES IN PHYSICS}

8. Weight standard (p. 22). The critic is wrong. ' $\mathrm{Kg}$ ' designates ambiguously a mass unit and a weight unit. The standard body at Sèvres, mentioned in my example of a referition (loc. cit.), happens to weigh $1 \mathrm{~kg}$, and also to 'mass' $1 \mathrm{~kg}$ - on the MKS system, that is. This ambiguity does not occur in alternative systems of units, such as the CGS system. Moral: conventions do not secure truth but they may foster confusion or prevent it.

Exercise: Make a semantic study of referitions (coordinating 'definitions') and find out what role, if any, they play in formalized physical theory.

9. Physical constants (p. 34). The critic is right. Boltzmann's constant $k$ is not a pure number but a number together with a certain dimension. More precisely (in the spirit of op. cit., p. 37), $k$ is a constant magnitude of a certain dimensional genus. What I wished to stress is that, unlike most other physical constants (like $c$ and $e$ ), $k$ is not a particular value of a function representing a definite property of a system: it is a nonreferential concept.

Problem: Provide a mathematical justification for the symbolic calculus of dimensions.

10. Scales arbitrary (p. 31). The critic is wrong. He entertains a mathematical notion of scale, not the usual physical concept I employ. A physical scale comes with a unit, whence I often call it a scale-cum-unit system. And, since units are arbitrary, scales are arbitrary as well. Further, any magnitude involving a given scale can be rescaled by a continuous isomorphism, i.e. by a function subject to the sole condition that it be continuous and operation-and-order preserving. A change of scale does not "change the edifice of physics" - as Strauss fears - if one rewrites the equations containing the affected magnitudes. In particular, the choice of an unusual time scale (like the logarithmic time scale proposed by Milne) would complicate our picture of the world but would not render it false. 
Exercise: Study the role of convention in theory and in experiment.

11. Michelson experiment (p. 193). The critic is wrong. Michelson did have two reference frames in mind: the Earth and the stationary (absolute) ether.

Exercise: Which of the formulas of SR can be subjected to fairly direct experimental tests? In particular, is the Lorentz transformation observable?

12. Mass in Newtonian physics (p. 210). The critic is wrong. If a gravitation theory fails to contain the concept of gravitational mass, either as primitive or as defined, and as different from the concept of mass tout court, then the popular 'law' of the equality of the gravitational and the inertial masses cannot even be stated in that theory. Hence the 'law' cannot be tested. (The famous measurements of Eötvös and of Dicke concern bodies with the same mass but different compositions.) If anyone claims to have produced a theory in which the two mass concepts appear as logically independent, let him exhibit such a theory and prove the independence of these concepts by Padoa's method. In any case, the existing theories make no room for the distinction, which is a child of operationalism (see [5]).

Exercise: Examine the way in which the mass and charge concepts are introduced (a) in elementary particle theories and (b) in geometrodynamics.

13. Mass in $S R$ (p. 201). The critic is wrong. Firstly, the law ' $E=m c^{2 \text { ' }}$ is not universal but restricted to SR mechanics. Indeed, it is derived in no theory but the latter; in particular, it does not occur in CEM, which was relativistic avant la lettre. Secondly, mechanics, whether classical or relativistic, does not happen to apply to the electromagnetic field, this being why special theories (among them CEM and QED) had to be devised. Thirdly, if the rest mass (or, more generally, the rest mass density) is set equal to zero in the equations of mechanics, as Strauss proposes, then nothing much remains - except the false statement that the force acting on the system concerned vanishes. To speak of zero mass bodies (e.g. particles) is to stretch the concept of body to the point of having it refer to the entire set of physical systems: pleasing as this would have been to Newton, we find it naively mechanistic. Thus take the 'general' equation ' $E^{2} / c^{2}=m_{0}^{2} c^{2}+p^{2}$ ' derived in SR mechanics. Setting $m_{0}=0$ in it does not yield ' $E=c p$ ', as my critic claims, but ' $E=0$ ', since by definition (in the context in which the formula holds) $p=m v=m_{0} v /\left(1-u^{2} / c^{2}\right)^{1 / 2}$. Of course, if that 'general' equation is torn off its context (SR mechanics) 
and applied to a photon, as Strauss and many a textbook do, by forgetting the meaning of ' $p$ ', then the formal expression ' $E=c p$ ' is indeed obtained. But this move is no better than applying the theory of evolution to photons, by redefining 'mutation' as a change in frequency and 'selection' as a filtering or absorption. Finally, the matter-field experiment invoked by the critic is irrelevant to my own example: while the former is a case of equilibrium, mine was one of non-equilibrium (temperature radiation built up in a cavity before the steady state is reached). In short, ' $E=m c^{2}$, applies only to bodies, and a body is, by definition, a physical system endowed with mass and a fairly definite localization.

Exercise: Trace the belief, popular among elementary particle physicists, that mass values are somehow (but only God knows how) determined by the type of interaction.

\section{A SUBSTITUTE FOR QM}

Strauss' main objection to my realist reinterpretation of QM is directed at my reintroducing, in a generalized way, de Broglie's original concept of probabilité de présence. He advocates replacing the concept of probability that a system $b e$ in a given state (which he calls prob ${ }_{1}$ ) by the probability of becoming, or making a transition from one state to another (his prob $_{2}$ ). In mathematical terms, Strauss proposes that we keep the concept of conditional probability while discarding the one of absolute probability. Now, the probability of a transition from state 1 to state 2 is, by definition, equal to the probability of state 2 (i.e. of the system being in state 2) given that the state 1 preceded it: $\mathbf{P}(1 \rightarrow 2)=\mathbf{P}(2 \mid 1)$. (See [9].) Hence there is no probability of becoming unless there is probability of being to begin with: prob $_{2}$ implies prob ${ }_{1}$ (but not conversely). If there are transitions among states, then except in the case of statistical equilibrium the occupation numbers of those states (which measure the probability of the system being in them) will change in time. Thus, imagine an amateur philosopher in either of two states: empiricist $(e)$ and dialectical $(d)$. Suppose his transition probabilities per unit time are $\mathrm{P}(e \mid d)=\frac{3}{4}$ and $\mathrm{P}(d \mid e)=\frac{1}{4}$. Assuming that the transitions are instantaneous (quantum jumps), our amateur philosopher will be thinking three-quarters of the time as a positivist and one-quarter as a dialectician. Consequently the probability of his being a positivist at an arbitrary instant equals $\frac{3}{4}$ while 
the probability of his being a dialectician is $\frac{1}{4}$. (This is, of course, an extreme idealization, for most of the time he would not think at all; it is also a classical model, for in QM he could be in a superposition of those two states, with the indicated weights.) Now to the criticisms of detail.

14. All the states are the same state (p. 246). The critic is right. My QM5(c) should indeed be scrapped. And the transformation properties of the state vector and the dynamical variables ('observables') should be added to the axiom group QM3. Also, the conditions under which a state vector and its transformed represent the same physical state should be adequately stated. One would then be able to get, as a metatheorem, the statement that QM - in particular its law statements - is covariant under unitary transformations.

Exercise: Perform the indicated operations.

15. Schrödinger equation (p. 247). The critic is wrong. In the formulation of QM I have chosen to axiomatize (the Schrödinger 'picture') the 'wave' (or rather pseudowave) equation is an equation proper - a partial differential equation in $t$ and $x$-hence one that, under certain restrictions, can be satisfied or solved. It is only in the alternative (and equivalent) Heisenberg 'picture' that the evolution of states is expressed as a unitary transformation. The critic's advice to scrap Schrödinger's equation would certainly bring happiness to all those who do not 'dig' QM, but it would leave us without a powerful tool for posing and solving problems in atomic and molecular physics.

Exercise: The two 'pictures' are mingled in the usual heuristic presentations of QM (e.g. Dirac's); show why they are different theories and list the advantages and disadvantages of each.

16. 'Pure' and 'mixed' nonsense (p. 247). The critic is right. My Dfs. 9 and 10 should be deleted. They play no role in the remainder of the book anyway.

Problem: Is the definition of a pure state for a space ensemble the same as the one for a time ensemble?

17. Q-densities (p. 246). The critic is wrong. It is odd to foist physical meanings on incomplete symbols (in Russell's sense), such as $\nabla$, as is usually done in regard to the quantum-mechanical operators. On the other hand there is nothing wrong with shifting the interpretation to the $Q$-densities $\psi^{*} Q \psi$, which are functions of position and time. Moreover, as Beck [1] has shown, in the relativistic theory those densities are welldefined geometric objects remarkably similar to the Maxwellian field and 
stress tensors. The formulas (1) and (2) invoked by Strauss actually reinforce the interpretation of $\psi^{*} Q \psi$ as a distribution function, since its space integral furnishes the distribution of the eigenvalues of $Q$. Surely the $Q$-densities occur only in the Schrödinger formulation of QM, but this is precisely the one I happened to be concerned with. If alternative formulations of QM (e.g. Heisenberg's) are far less convenient for computation and also more difficult to assign physical meanings to (except by analogy to classical mechanics), so much the worse for them.

As to Strauss' novel proposal to regard formula (2) in his review as "the correct semantical postulate", it would not do: $\left|\left(\psi, \varphi_{k}\right)\right|^{2}$ is not interpretable as the probability of a physical transition, i.e. as a transition probability. Indeed, there is no transition at the back of that expression, which derives from a purely mathematical decomposition of the state vector into the eigenstates of an arbitrary operator $Q$ which need not even give rise to a physically meaningful $Q$-density. The number $\left|\left(\psi, \varphi_{k}\right)\right|^{2}$ is nothing but the statistical weight, or probability (or propensity) of the $k$ th eigenvalue $q_{k}$ of $Q$. In this point my interpretation agrees with the usual one. It is only when thinking in the Copenhagen fashion (hard line), i.e. when associating a (phony) apparatus with every quantum-mechanical formula, that Strauss' proposed reinterpretation becomes natural, for then the line between real transitions and imaginary ones is effaced. But since no mention of any apparatus is made in most of the formulas of QM (once freed of their philosophical deadwood), Strauss' transition probabilities are (unlike my $Q$-densities) definitely ghostly, as ghostly as the ever-present apparatus - the ghostliness of which has been shown elsewhere [6]. Interpretations should not be arbitrary: at the very least they should respect the structure of the interpreted symbol and they should cohere with the interpretations assigned to related symbols.

Finally, Strauss' advice to axiomatize QM without specializing either the formulation or the representation (two concepts which, incidentally, he fails to distinguish) is interesting. So much so, in fact, that I will leave this task to him. With two cautions, though. First: the theory is to be axiomatized rather than 'illustrated' (distorted) by a discussion of phony (imaginary) experiments, in the spirit of operationalism (as is the case with Strauss' own 'Restatement of QM' [12]). Second: while the freeing from a particular formulation (e.g. Schrödinger's) and a particular representation (e.g. the position representation) is mathematically interesting, 
because it yields a more general and abstract theory it is harder to interpret and apply. (Likewise, for foundations purposes it is convenient to work in Cartesian coordinates rather than in the more general curvilinear coordinates, which are less perspicuous and introduce what I call spurious quantizations. Maximal generality is not always to be preferred in foundations research, which is concerned not only with form but also with content.) For that reason the working physicist - the one who solves actual problems rather than playing with gedankenexperiments - prefers to work in a particular formulation and in a specific representation.

Problem: Seek to divide the basic 'quantities' of QM into manifest (actual) and dispositional (potential) and see how the actualist programme (general reduction of dispositionals to actuals) fares in QM. Hint: position is an actual property in CM and no property at all in QM, while the position density $\psi^{*} x \psi$ is a dispositional property.

\section{SHORTCOMINGS}

18. Two problems not touched on in 'Foundations of Physics'. Irrelevant. Anyone can make a far longer list of problems that are not tackled in this book, or for that matter in any other book. Hardly a shrewd comment in view of the finite length of real books. Only critics are infinite.

19. Intertheory relations. Irrelevant. Again, this is one of the many points that could only be alluded to in the book. Yet, unlike the usual presentations of physical theories, mine at least mentions the theories presupposed by every physical theory. I deal with intertheory relations in a longish article to be published jointly with Dr. Strauss' own paper on the subject [7].

20. Pragmatic aspects of physical theory. Irrelevant. It is like complaining, about a treatise on theoretical mechanics, that the tests and practical applications of that theory have been overlooked by its author. After all, my book was on the foundations of physics. Besides, I have discussed elsewhere the philosophy of tests and applications of scientific theories [8]. Anyway, the pragmatic aspect of theorizing is the one that gets most of the attention in the usual treatments, at the expense of the logical and semantical aspects. The pragmatic interpretation (as different from the semantic one) of theoretical formulas is treated in another paper of mine [6]. 
This brings my rejoinder to an end. Rather than closing with a moral I should like to end with an invitation. Foundations research being, like science and exact philosophy, a collective enterprise, is best advanced by avoiding personal attacks and working together in a scholarly and constructive spirit. Let us then address ourselves to problems rather than to individuals, and let us criticize ideas instead of attacking persons.

\section{ACKNOWLEDGMENTS}

I am grateful to Dr. Strauss for having pointed out five of the errors that occur in Foundations of Physics and to Professor Hintikka for having given me the opportunity of acknowledging those errors and upgrading thirteen pseudomistakes. My thanks are also due, for sharp yet constructive and friendly criticism, to Professor Erhard Scheibe (Göttingen) and to my student Mr. Hart Katz.

McGill University, Montreal

\section{BIBLIOGRAPHY}

[1] Beck, G., 'Field Concepts in Quantum Theory', Reviews of Modern Physics 17 (1945) 187-194.

[2] Bunge, M., Foundations of Physics, Berlin/Heidelberg/New York 1967.

[3] Bunge, M., 'Laws of Physical Laws', American Journal of Physics 29 (1961) 518-529.

[4] Bunge, M., 'Physical Time: the Objective and Relational Theory', Philosophy of Science 35 (1968) 355-388.

[5] Bunge, M., 'On Mach's Nonconcept of Mass', American Journal of Physics 36 (1968) 167-168.

[6] Bunge, M., 'What are Physical Theories About?', American Philosophical Quarterly, Supplementary Monograph No. 3 (1969).

[7] Bunge, M., 'Problems Concerning Intertheory Relations', in Proceedings of the 1968 Salzburg Colloquium in Philosophy of Science, forthcoming.

[8] Bunge, M., Scientific Research, vol. II, Berlin/Heidelberg/New York 1967.

[9] Feller, W., An Introduction to Probability Theory and its Applications, 2nd ed., New York and London 1957.

[10] Khintchine, A. I., 'La méthode des f onctions arbitraires et la lutte contre l'idéalisme dans la théorie des probabilités', in Questions Scientifiques, tome V, Paris 1954.

[11] Strauss, M., 'Corrections to Bunge's "Foundations of Physics" (1967)', Synthese 19 (1968-69) 433-442.

[12] Strauss, M., 'Intertheory Relations', in the volume cited in [7]. 